\title{
Response of Bacterial Communities upon Application of Different Innovative Organic Fertilizers in a Greenhouse Experiment Using Low-Nutrient Soil Cultivated with Cynodon dactylon
}

\author{
Marina Zanardo ${ }^{1}$, Riccardo Rosselli ${ }^{2}$, Andrea Meneghesso ${ }^{3}$, Gaurav Sablok ${ }^{4}$, \\ Piergiorgio Stevanato ${ }^{1}$, Marion Engel ${ }^{5,6}$, Adriano Altissimo ${ }^{7}$, Lisanna Peserico ${ }^{7}$, \\ Valentina Dezuani ${ }^{7}$, Giuseppe Concheri ${ }^{1}$ (D), Michael Schloter ${ }^{5}$ and Andrea Squartini ${ }^{1, *}$ \\ 1 Department of Agronomy, Food, Natural Resources, Animals, and Environment-DAFNAE, \\ University of Padova, Viale dell’Università 16, 35020 Legnaro (Padova), Italy; \\ marinazanardo1@gmail.com (M.Z.); stevanato@unipd.it (P.S.); giuseppe.concheri@unipd.it (G.C.) \\ 2 Division de Microbiología, Universidad Miguel Hernandez, 03202 San Juan de Alicante, Spain; \\ rrosselli@umh.es \\ 3 Department of Biology, University of Padova, Via Ugo Bassi 58b, 35131 Padova, Italy; \\ andrea.meneghesso@unipd.it; \\ 4 Department of Biodiversity and Molecular Ecology, Research and Innovation Centre, Fondazione Edmund \\ Mach, Via E. Mach 1, San Michele all'Adige, 38010 Trento, Italy; sablokg@gmail.com \\ 5 Research Unit for Comparative Microbiome Analysis, Helmholtz Zentrum München, \\ Ingolstädter Landstraße 1, 85764 Neuherberg, Germany; marion.engel@helmholtz-muenchen.de (M.E.); \\ schloter@helmholtz-muenchen.de (M.S.) \\ 6 Research Unit Scientific Computing, Helmholtz Zentrum München, Ingolstädter Landstraße 1, \\ 85764 Neuherberg, Germany \\ 7 Landlab Studio Associato, Via Quintarello 12A, 36050 Quinto Vicentino (Vicenza), Italy; \\ a.altissimo@landlab.net (A.A.), l.peserico@landlab.net (L.P.); v.dezuani@landlab.net (V.D.) \\ * Correspondence: squart@unipd.it; Tel.: +39-0498272923; Fax: +39-049-8272929
}

Received: 30 July 2018; Accepted: 4 September 2018; Published: 6 September 2018

\begin{abstract}
Assessing the response of microbial communities to nutrient inputs in man-managed soils is of primary importance to understand the impact on ecosystem services provided by the soil microbiome. In this study, a low-nutrient soil was supplemented with seven different innovative fertilizers including matrixes of plant, animal, fungal or synthetic origin, and dosed to deliver the same amount of nitrogen. Growth of a potted grass crop (Cynodon dactylon) was recorded and the fertilizers were scored by the plant yield obtained in a greenhouse study. Soil was sampled at 9 and 58 days after the addition and bacterial community composition was analyzed after soil DNA extraction through pyrosequencing of $16 \mathrm{~S}$ rDNA gene amplicons. Over 900 bacterial genera were detected, belonging to 21 described and 19 candidate phyla. In spite of the equal dose of nitrogen delivered, specific groups were fostered by given fertilizers; in particular marked effects on some phyla were displayed by a yeast-based fertilizer, which was also most effective in plant productivity. The main shifts were observed shortly after the fertilizer application, followed by a gradual stabilization of the equilibrium and by a rise in community evenness.
\end{abstract}

Keywords: organic fertilizers; bacterial communities; $16 \mathrm{~S}$ amplicons

\section{Introduction}

Soils are among the most heterogeneous ecosystems on Earth, and in spite of unfavorable conditions in terms of nutrient availability and predation, thousands of microbial species find their primary habitat 
in them. Estimates indicate that one gram of soil may harbor between $2 \times 10^{3}$ and $8.3 \times 10^{5}$ different species [1,2]. Currently, surveys and counts rely on high throughput metagenomic approaches [3-8].

Bacteria, archaea and fungi adapted to live in soil can regulate plant growth and health through their direct role in processes, such as organic matter decomposition, nutrient cycling and pathogen control. The structure of soil microbial communities thus entails a special importance for its primary productivity. A better understanding of the mechanisms regulating soil life and functionality in response to external inputs in agriculture is fundamental to improve soil management, and in particular nitrogen $(\mathrm{N})$ requirement and fate in agriculture, and to reduce the threats to the environment, and in turn to human health.

$\mathrm{N}$ inputs to soil can have important impacts on the environment, and many studies have focused on their effects on plant growth, $\mathrm{N}$ and carbon cycles, and emissions of greenhouse gases to the atmosphere [9-12]. Less well understood are the impacts of fertilizer inputs on soil bacterial communities, even though bacteria represent a major portion of living biomass in terrestrial ecosystems [13] and their activities are intimately tied to belowground processes. Some studies have reported shifts of bacterial community composition in response to $\mathrm{N}$ amendments or particular fertilization practices [14-19].

The aim of the present work was to detect specific responses of various bacterial groups in the presence of a series of innovative fertilizers, and to compare their effects on soil bacterial communities over a two-month period. The innovative aspect in the products used consists in the fact that these fertilizers are novel mixtures recently set up by the ILSA S.p.A., Arzignano VI, Italy, and recycle different agricultural or industrial byproducts, whose properties on plants are under investigation.

The working hypothesis of the present investigation was that, notwithstanding the equal level of $\mathrm{N}$ delivered, different microbial groups would be affected depending on the fertilizer composition. This rests on the assumption that, although $\mathrm{N}$ is a major limiting nutrient for soil life, microbial communities would respond differently, depending on the form in which it is supplied. For this reason, the $\mathrm{N}$ dosage was set equal in all fertilizers. To frame this rationale, it needs to be considered that organic carbon, along with $\mathrm{N}$, can be a strong determinant of microbial population changes. In our fertilizers, we have chosen a series in which organic carbon is the variable and an equal amount of $\mathrm{N}$ is bound to it in different ways (the amino group within peptides in the organic fraction or bound to carbonyl in urea or as the heterocyclic ring with $\mathrm{N}$ in Oxy-Amino-Triazine (OATr)). The microbial proficiency in extracting nitrogen from the different Carbon-based substrates and exploiting it structurally for their detectable fluctuation dynamics is, in this respect, the phenomenon under study.

A deeper understanding of these aspects, along with the corresponding plant productivity responses, will allow to plan improved fertilization strategies to exploit the plant-soil-microbe continuum in its ideal balance conditions for plant growth promotion.

The fertilizers included products of biological origin as Thermal-Hydrolyzed-Leather (THL), Castor Cake (CC), Neem (Nm), and Yeast-based fertilizer $(\mathrm{Y})$, their combinations with urea consisting in: Urea-THL-Yeast (UTY) and Urea-THL-Neem (UTN), as well as the synthetic fertilizer OATr. The fertilizers were tested on an agricultural low-nutrient soil, in a greenhouse study using the creeping perennial grass Cynodon dactylon (C. dactylon) as the model plant species. Bacterial diversity was analyzed via deep 454 sequencing of $16 \mathrm{~S}$ rDNA amplicons obtained from extracted DNA from soil samples taken 9 days and 58 days after fertilizer application. Soil sampling time points were selected based on our previous study, which made use of the same set up. In that study, the abundance of genes involved in the $\mathrm{N}$ cycle was measured by quantitative polymerase chain reaction (qPCR) and indications were obtained that, 9 days after application, compositional shifts are clearly detectable, whereas 58 days after application, a stabilization and partial reversion to the original conditions is observed [20]. 


\section{Material and Methods}

\subsection{Greenhouse Fertilization Trial}

A greenhouse trial was set up in Summer 2012 with the aim to compare the effects of different fertilizers on plant growth and soil bacterial communities. The fertilization experiment included 8 treatments (one unfertilized control and seven fertilizers). Each treatment was replicated 4 times in a randomized design, resulting in overall 32 pots. The names and the descriptions of the fertilizers, which were all provided by a commercial supplier, (ILSA S.p.A., Arzignano, Vicenza, Italy) are given in Table 1. In order to minimize nutrient background and to put in evidence the effects of the different organic fertilizers, the soil used was a mixture of sand (50\%) and a low fertility soil which had been purposely impoverished in $\mathrm{N}$ through several years of repeated monoculture cropping with cereals, without any fertilization. The soil $\mathrm{pH}$ value was $7.6\left(\mathrm{CaCl}_{2}\right), 8.1\left(\mathrm{H}_{2} \mathrm{O}\right)$ and its $\mathrm{C} / \mathrm{N}$ ratio was 13.7. Further detailed chemical and physical properties of the soil used for the test have been previously reported [20].

The pots had a diameter of $20 \mathrm{~cm}$, and contained $5.5 \mathrm{~kg}$ of soil. The fertilizers were mixed in the upper $5 \mathrm{~cm}$ of the substrate, and subsequently a sod with pre-grown $C$. dactylon was placed on the top.

Fertilizers were added to obtain a final $\mathrm{N}$ input corresponding to $150 \mathrm{~kg} / \mathrm{ha}$. Moreover, $\mathrm{P}_{2} \mathrm{O}_{5}$ and $\mathrm{K}_{2} \mathrm{O}_{5}$ were added to deliver $50 \mathrm{~kg} / \mathrm{ha}$ and $100 \mathrm{~kg} / \mathrm{ha}$, respectively. The fertilization trial lasted for 2 months, during which plant growth measurements and soil sampling were done.

Watering was carried out by capillary infiltration from the bottom of the pots by keeping a constant level of water in plastic planter saucers placed under each pot. Refilled water dosage corresponded to the daily restitution of evapotranspiration, which spanned between $3 \mathrm{~mm}$ and $5 \mathrm{~mm}$. The mean temperature in the greenhouse across the trial period was $26.7^{\circ} \mathrm{C}$. The light regime was the natural daylight span occurring at coordinates 45_34007.9800 N; 11_37014.6300 E from mid-June to mid-August (15 h $40^{\prime}$ and 14 h 5').

Plant growth in terms of height, fresh weight and dry weight (after cutting at the ground level) of $C$. dactylon were measured in all treatments three times during the two months period, at days 20 , 35 and 56 after fertilization.

Table 1. Fertilizers used in the greenhouse trial. The contents in macronutrients are indicated.

\begin{tabular}{|c|c|c|c|c|c|c|}
\hline Abbreviation & Name & N (\%) & $\mathrm{C}(\%)$ & $\mathrm{P}_{2} \mathrm{O}_{5}(\%)$ & $\mathrm{K}_{2} \mathrm{O}(\%)$ & $\mathrm{C} / \mathrm{N}$ \\
\hline THL & Thermal-Hydrolyzed-Leather & 12.8 & 41.2 & $<0.1$ & 0.0 & 3.2 \\
\hline Y & Yeast & 6.1 & 43.2 & 1.3 & 1.7 & 7.1 \\
\hline $\mathrm{Nm}$ & Neem & 3.5 & 42.0 & $0.5-1.0$ & $1.0-2.0$ & 12 \\
\hline $\mathrm{CC}$ & Castor Cake & 4.3 & 45.0 & $2.0-2.5$ & $1.0-1.5$ & 10.5 \\
\hline OATr & Oxy-Amino-Triazine & 50.4 & 32.4 & 0.0 & 0.0 & 0.6 \\
\hline UTY & Urea-THL-Y & 21.5 & 35.0 & 0.1 & 0.2 & 1.6 \\
\hline UTN & Urea-THL-N & 21.1 & 35.0 & $0.15-0.25$ & $0.2-0.4$ & 1.7 \\
\hline
\end{tabular}

\subsection{Soil Sampling and DNA Extraction}

Soil was sampled with a 1.5-cm-diameter corer at a 5-cm depth in the center of the pots under the C. dactylon sod, 9 and 58 days after the addition of the fertilizers. Sixty-four samples were taken for amplicon pyrosequencing, representing 4 biological replicates of 8 treatments at two sampling times. After sampling root portions were discarded if present and $2 \mathrm{~g}$ of soil were immediately frozen in liquid nitrogen, and then stored at $-80{ }^{\circ} \mathrm{C}$ until the extraction of nucleic acids.

Nucleic acids were extracted from $0.45 \mathrm{~g}$ of thawed and air-dried soil. Soil was added to a Lysing Matrix E tube (MP Biomedicals, Santa Ana, CA, USA), with $750 \mu \mathrm{L}$ of $120 \mathrm{mM} \mathrm{NaPO}_{4}$ buffer (pH 8) and $250 \mu \mathrm{L}$ of TNS (500 mM Tris- $\mathrm{HCl} \mathrm{pH} 8.0,100 \mathrm{mM} \mathrm{NaCl}, 10 \%$ SDS $w / v)$. To perform cell lysis, the tube was shaken $30 \mathrm{~s}$ at $5.5 \mathrm{~m}$ per second in a bead beater (Precellys Bead Beater, Bertin Technologies, Montigny-le-Bretonneux, France). Soil and cell debris were pelleted by centrifugation at $13,200 \times g$ at $4{ }^{\circ} \mathrm{C}$ for $20 \mathrm{~min}$, and the supernatant was transferred to a $2-\mathrm{mL}$ fresh RNase-free tube. Nucleic 
acids were extracted and purified with phenol/chloroform/isoamylalcohol (25:24:1, pH 8, v/v) and subsequently with chloroform/isoamylalcohol $(24: 1, v / v)$. The obtained aqueous phase was mixed with 2 volumes of PEG solution ( $30 \% w / v$ polyethylene glycol 6000 in $1.6 \mathrm{M} \mathrm{NaCl})$, incubated at $4{ }^{\circ} \mathrm{C}$ for $2 \mathrm{~h}$ and centrifuged at $13,200 \times g$ at $4{ }^{\circ} \mathrm{C}$ for $15 \mathrm{~min}$. The nucleic acid pellet was washed with $70 \%$ ethanol, air-dried and resuspended in $50 \mu \mathrm{L}$ (DEPC)-water. All steps were performed on ice. DNA was quantified spectrophotometrically on a Nanodrop (Thermo Scientific, Tewksbury, MA, USA). DNA samples were stored at $-80^{\circ} \mathrm{C}$.

\section{3. $16 \mathrm{~S}$ rRNA Gene Barcoded Amplicon Sequencing}

Amplicon pyrosequencing was performed on a 454 GS FLX Titanium system (Roche, Penzberg, Germany) under the following operating conditions. Barcoded amplicons for multiplexing were prepared using the primers Ba27f (5'-AGA GTT TGA TCM TGG CTC AG-3') and Ba519r (5'-TAT TAC CGC GGC KGC tg-3') [21] extended with the respective A or B adapters, key sequence and multiplex identifiers (MID) as recommended by Roche. As advised by Berry and co-workers [22], a two-step PCR approach was selected, with the addition of the MID-primers only in the second PCR amplification, to avoid biases and increase reproducibility. Each amplification was done in triplicate. The first PCR was performed with the following cycling conditions: initial denaturation $\left(94{ }^{\circ} \mathrm{C}, 10 \mathrm{~min}\right)$, followed by 22 cycles of denaturation $\left(94^{\circ} \mathrm{C}, 30 \mathrm{~s}\right)$, annealing $\left(52{ }^{\circ} \mathrm{C}, 30 \mathrm{~s}\right)$ and extension $\left(72{ }^{\circ} \mathrm{C}, 60 \mathrm{~s}\right)$ and by a final extension step at $72{ }^{\circ} \mathrm{C}$ for $10 \mathrm{~min}$. Each $25-\mu \mathrm{L}$ PCR reaction contained 1x PCR buffer (containing $\mathrm{MgCl}_{2}$ ), $0.2 \mathrm{mM}$ dNTPs, 1.25 U Fast Start HiFi Polymerase (Roche, Penzberg, Germany), $0.24 \mathrm{mg} / \mathrm{mL}$ BSA, $0.25 \mathrm{mM}$ of each primer and $1 \mu \mathrm{L}$ of template DNA (at a concentration of $20 \mathrm{ng} / \mu \mathrm{L}$ ). The second PCR was performed with the same cycling conditions as the first, but with only 6 cycles of denaturation, annealing and extension. Each $25-\mu L$ PCR reaction contained 1x PCR buffer (including $\mathrm{MgCl}_{2}$ ), $0.2 \mathrm{mM}$ dNTPs, $1.25 \mathrm{U}$ Fast Start HiFi Polymerase (Roche), $0.24 \mathrm{mg} / \mathrm{mL}$ BSA, $0.25 \mathrm{mM}$ of each MID-primer (Biomers Pte Ltd., Ulm, Germany) and $5 \mu \mathrm{L}$ of the previous PCR product. Amplicons were purified using Agencourt AMPure XP beads (Beckman Coulter, Brea, CA, USA), and quantified with the Quant-iT PicoGreen dsDNA quantification kit (Invitrogen, Thermo Fisher Sciebntific Inc. Monza, Italy). The purification was checked using an Agilent Bioanalyzer 2100 (Agilent Technologies, Santa Clara, CA, USA). Amplicons were diluted to $10^{9}$ molecules/ $\mu \mathrm{L}$ and equimolarly pooled in 4 pools of 16 samples.

Emulsion PCR, emulsion breaking and sequencing were performed applying the GS FLX Titanium chemistry following the supplier's protocol.

\subsection{Data Analysis and Statistics}

Raw sequence analyses were conducted using the QIIME pipeline [23]. About 450,000 raw sequences were generated by the run; the subsequent procedure of quality filtering and barcode assignment involved separate denoising with a pre-processing step by using split_libraries.py at recommended QIIME settings (-w 50 -g combination).

After such filtering steps and chimera removal, about 246,000 good-quality sequences were used for further analyses, with a mean of about 3800 sequences per sample. Phylotypes were selected at a $97 \%$ sequence similarity level and the taxonomic identity was determined using the Ribosomal Database Project (RDP) database scheme. Sequence data have been deposited at the European Biotechnology Institute (EBI) database (http:/ /www.ebi.ac.uk/) and are available under the code PRJEB21947.

The QIIME software (version 1, http: / / qiime.org/) was also used to calculate and draw rarefaction curves based on the number of observed species and the Shannon index.

The relative abundance data of each taxon were used for further analysis, to compare different soil samples and to deduce individual effects caused by different fertilizers on soil bacterial communities.

Diversity indices (Simpson index 1-D and Evenness $\mathrm{e}^{\wedge} \mathrm{H} / \mathrm{S}$ ) were calculated using the Past Software (Paleontological Statistics Software Package, version 2.12); after normality distribution 
evaluation, statistical differences were analyzed with $\mathrm{T}$ tests for paired samples (normally distributed data) or the non-parametric Wilcoxon test (non-normally distributed data).

\section{Results}

\subsection{Plant Growth}

In order to evaluate how the presence of different fertilizers affected plant growth, three grass cuts were carried out during the two-month incubation. Height, fresh weight and dry weight of grown C. dactylon were measured. The cumulative data are shown in Table 2 . The significance of differences was ascertained by an ANOVA analysis, of which results are shown in Table S1 in Supplementary Materials.

The fertilizers CC and Y promoted plant growth most, followed by THL and by the fertilizers UTY and UTN. These last two, which are composite fertilizers, and only differ in their organic vegetable part $(10 \% \mathrm{Y}$ and $20 \% \mathrm{Nm}$, respectively), promoted plant growth to a very similar extent. N and OATr, which are slow release fertilizers and are meant to decrease the loss of $\mathrm{N}$ in the environment through nitrification and denitrification, had lower effects on plant growth. In particular, the yield in the presence of OATr was very similar to that observed in the non-fertilized control soil.

Table 2. Cumulative results for the 3 considered plant growth parameters (Mean \pm SD). THL: Thermal-Hydrolyzed-Leather; Y: Yeast; Nm: Neem; CC: Castor Cake; UTY: Urea-THL-Yeast; UTN: Urea-THL-Neem; OATr: Oxy-amino-triazine; Ctr.: unfertilized control. Significance of differences (ANOVA) for each of the parameters is reported in Table S1 in Supplementary Materials.

\begin{tabular}{cccc}
\hline \multicolumn{4}{c}{ Plant Growth Measurements } \\
\hline Fertilizer & $\begin{array}{c}\text { Cumulative Average } \\
\text { Height }(\mathbf{c m})\end{array}$ & $\begin{array}{c}\text { Cumulative Average } \\
\text { Fresh Weight } \mathbf{( g )}\end{array}$ & $\begin{array}{c}\text { Cumulative Average } \\
\text { Dry Weight (g) }\end{array}$ \\
\hline THL & $12.80 \pm 0.83$ & $70.21 \pm 7.31$ & $20.09 \pm 1.84$ \\
Y & $16.51 \pm 1.70$ & $96.21 \pm 8.62$ & $26.24 \pm 1.26$ \\
Nm & $12.06 \pm 1.19$ & $57.72 \pm 4.85$ & $17.11 \pm 1.68$ \\
CC & $16.28 \pm 1.60$ & $96.74 \pm 4.05$ & $26.49 \pm 0.97$ \\
UTY & $13.86 \pm 0.70$ & $74.86 \pm 6.75$ & $20.97 \pm 2.37$ \\
UTN & $13.91 \pm 1.27$ & $71.47 \pm 7.87$ & $21.03 \pm 1.68$ \\
OATr & $10.16 \pm 0.68$ & $26.23 \pm 2.94$ & $9.32 \pm 0.88$ \\
Ctr. & $9.25 \pm 0.82$ & $25.67 \pm 5.23$ & $8.89 \pm 1.55$ \\
\hline
\end{tabular}

\subsection{Composition of Soil Bacterial Communities}

The rarefaction analysis at a 95\% similarity, aiming at genus level (Supplementary Materials, Figure S1A), highlighted the presence of complex soil bacterial communities. The shape of the rarefaction curves (not asymptotic) indicated, as typical for soil community samples which harbor a very high taxa richness, that the sequencing effort was not high enough to cover complete bacterial diversity, but almost all curves were nevertheless in the range of $80-90 \%$ saturation. The two samples with a somewhat lower number of observed species represent two of the four replicates of the soil treated with $Y$, sampled at day 9. The rarefaction curves relative to the Shannon diversity index (Supplementary Materials, Figure S1B) show nonetheless a satisfactory coverage of such parameters for all samples.

Overall 900 bacterial genera were detected, belonging to 21 described and 19 candidate phyla (Table S2, Supplementary Materials). About one third (296 genera) were members of the phylum Proteobacteria, consistent with a marked rhizosphere influence in composition. Among the five classes of Proteobacteria detected in our samples, Alphaproteobacteria showed high levels of diversity (H index), whereas for Epsilonproteobacteria, just one OTU (operational taxonomic unit) was detected. For Actinobacteria, 123 genera were detected, while 110 were found for Firmicutes. Acidobacteria presented about four times less bacterial genera when compared to Actinobacteria, indicating a low diversity within this bacterial phylum, which was confirmed by the calculation of diversity indices. 


\subsection{Effects of the Fertilizers on Soil Bacterial Communities}

Major effects on the composition of soil bacterial communities caused by the application of different kinds of fertilizers could be highlighted describing the relative abundance of the main bacterial phyla (Figure 1). Proteobacteria were highly abundant in all the treated soils and encountered between $22 \%$ (in soil treated for 9 days with Y) and $40 \%$ (in soil treated for 9 days with UTY). More evident differences could be seen for Firmicutes, which accounted for $5-15 \%$ of the bacterial community in the unfertilized soil as well as in the two composite urea-based fertilizer-treated soils (UTY and UTN). In contrast, they were far more abundant (up to $45 \%$ ) in soils amended with the fertilizers Y, THL, CC, and Nm. Their lowest relative abundance was detected in soils fertilized with OATr (4-8\%). Results for Acidobacteria and Actinobacteria were comparable: In the presence of the four single-matrix fertilizers, and in particular with $Y$, their numbers in soils were lower when compared to the unfertilized soil, while with OATr, UTY and UTN, their relative abundance did not seem to be influenced.

THL

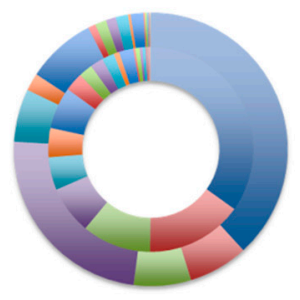

$\mathrm{Nm}$

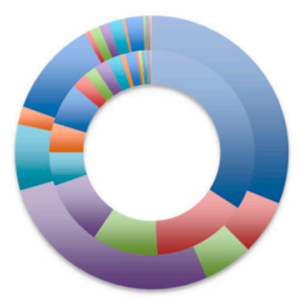

UTY

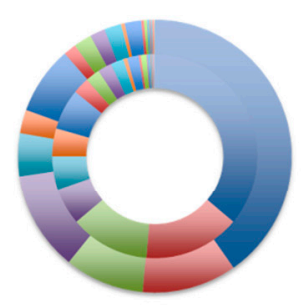

Y

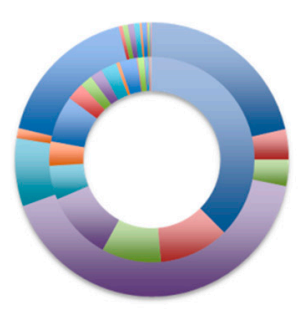

CC

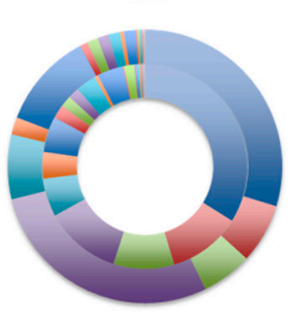

OATr

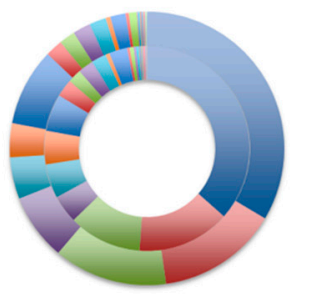

UTN

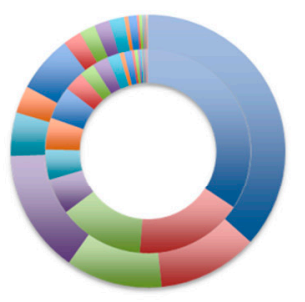

Ctr

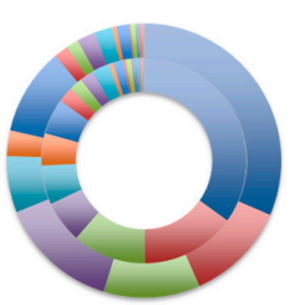

- Proteobacteria

- Acidobacteria

$=$ Actinobacteria

a Firmicutes

ఐ Unassigned;Other

a Chloroflexi

$=$ Bacteroidetes

= Gemmatimonadetes

Nitrospirae

= Planctomycetes

= Verrucomicrobia

$\triangle$ Armatimonadetes

$=$ Cyanobacteria

aWS3

- TM7

$\cong \mathrm{OD} 1$

= Fibrobacteres

= TM6

Elusimicrobia

[Thermi]

Tenericutes

- GNO2

$=\mathrm{BRC} 1$

OP3

= Spirochaetes

a Chlorobi

WS4

= OP11

a Other

a SR1

Inclassified

$=$ GNO4

FBP

- NKB19

BHI80-139

GAL15

MVP-21

WPS-2

WS2

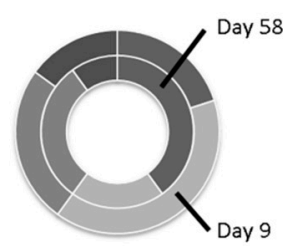

Figure 1. Double concentric pie charts showing the changes in soil bacterial communities at the phylum level over time. Outer circle: sampling at Day 9; inner circle: sampling at Day 58. The identities of annotated phyla, starting from each circle top and proceeding clockwise, can be read upon descending along on the list on the right. 
Bacteroidetes were more abundant in soils treated with Y. The apparent abundance of Chloroflexi seemed to be slightly impaired by fertilization with matrixes of biological origin as they were more frequent in the unfertilized soil and in the soil fertilized with OATr, than in all the other treatments. Other phyla were present at percentages lower than $2 \%$ in all the samples. Overall OATr promoted the relative increase of many low-abundance phyla. With the fertilizer $Y$, on the other hand, many phyla, both abundant and low-number taxa, showed lower values while few different taxa appeared in increased proportions.

\subsection{Effect of the Sampling Time Point on Soil Bacterial Communities}

Diversity indices indicated no differences between treatments, when considering the same sampling day; however, more evident changes were found when the sampling time point was taken into account (Figure 2). In particular, at day 58, both richness and evenness increased. Having verified the non-normality of the data distribution, the non-parametric Wilcoxon Test was chosen to express the statistical significance of the interactions between the diversity indices and the sampling day. Differences ( $p$-values $<0.05)$ resulted for Evenness $(p<0.000542)$ and for the Simpson diversity index $(p<0.021974)$.

Overall, the composition of soil bacterial communities in fertilized soils changed over the experimental period of two months. Changes at the phylum level can be followed in Figure 1, where the double pie charts allow the direct comparison of the relative abundance of each bacterial phylum at day 9 and at day 58 .

The relative abundance of Acidobacteria increased in all the soil samples, except for OATr, where they remained stable. In general, Bacteroidetes and Firmicutes, which were very abundant at day 9, showed a relative decrease over time. This was particularly evident in soils treated with $Y$, where Firmicutes DNA was present at a significantly higher level (45\%) at day 9 when compared to all other treatments (from $8 \%$ in OATr to $31 \%$ in CC). Fertilization with Y also caused a change in the relative number of Proteobacteria (from $22 \%$ to $38 \%$ ), which remained stable with the other treatments. Less abundant phyla showed higher figures passing from day 9 to day 58 .

The analysis of changes at lower hierarchical ranks down to the genus level can be followed in Table S3 (Supplementary Materials), where the ratios of taxa percentages between day 58 and day 9 are reported. The data used are the means of the four replicates.

To carry out this analysis, we first ran a repeated measurement with ANOVA for correlated samples to identify which taxa were showing relative changes to a statistically significant extent across treatments and sampling times. The 4 replicates of each condition were taken into account separately as groups. From this analysis, 109 taxa were selected, which are compared in Table S3 (Supplementary Materials). In that table the frequency observed at day 58 is divided by that recorded at day 9. Values $<1$ do, therefore, indicate decreasing frequencies and vice versa for values $>1$. Background colors of the table cells, with shades from red to green, highlight such trends, respectively. Overall shifts towards a lower relative abundance at day 58 are obvious for Bacteroidetes and Firmicutes. Among the latter, Clostridia and Bacilli were the most abundant classes, and the relative decrease over time of Clostridia was particularly evident. From the other side, positive successional dynamics outcomes appeared for Acidobacteria, Actinobacteria and Proteobacteria with the exception of Betaproteobacteria, Epsilonproteobacteria and of the genus Enterobacter.

The relative abundances of some Proteobacteria, being of particular interest in agriculture, were differently influenced over time by amendment with different fertilizers (Supplementary Materials, Table S3). For example, the N-fixer Azospirillum appeared more represented at day 9 in soils treated with $\mathrm{Y}$ and $\mathrm{CC}$, which were also the best performing fertilizers in terms of plant yield (Table 2). In general, genomes of members of the order Rhizobiales appeared relatively more abundant at day 58 . The fertilizers that mostly enhanced the relative frequency of Rhizobiales were also those that promoted higher plant yields (CC, Y, THL). The nitrifier Nitrospirae increased in relative abundance over time in all the samples, 
except for UTY, where it remained stable. The taxon showed a pattern of relative doubling during the nearly 50 examination days.

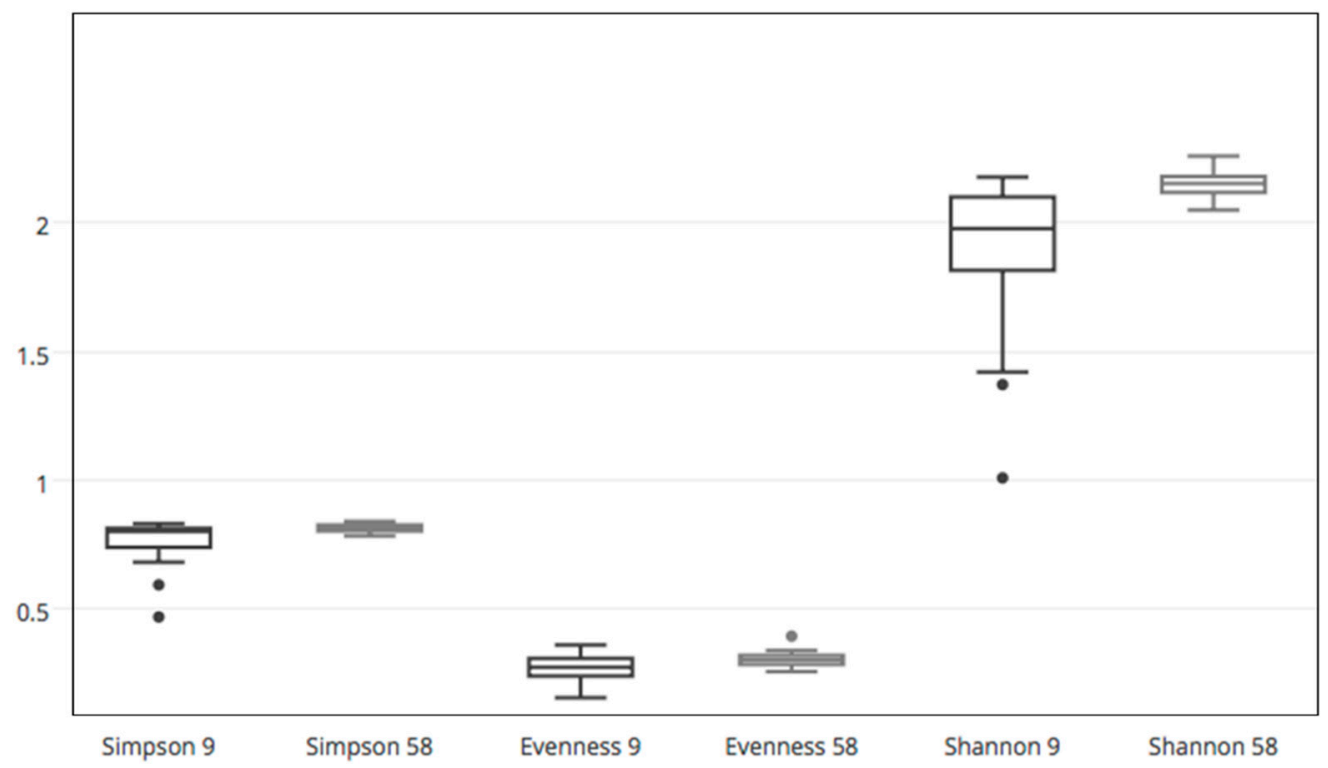

Figure 2. Boxplots of the three diversity indices at the two sampling times.

\section{Discussion}

The analysis revealed a considerable extent of diversity within the chosen soil, in spite of its sandy texture and low nutrient content. Rarefaction analysis showed that a higher number of sequences would be required to approximate the number of OTUs in the considered soils, and this kind of situation was also reported in other works [18]. Schloss and Handelsman [1] suggested that as many as 18,000 sequences per sample should be required to fully describe soil community diversity. However, this was not the main goal of this study, as the focus here was on the detection of the major relative changes in soil microbial communities induced by the presence of a certain fertilizer.

As regards the expected scenarios in this kind of analysis, extensive studies focusing on the impact of manure or chemical fertilizers in arable soils report that the application of those could significantly change the soil bacterial community [8]. The phenomenon can be neither general nor permanent, as some authors demonstrated that specific soil taxa as Pseudomonas could be temporarily affected, depending on the type of organic fertilizer [5].

The major phyla detected in our soil-Proteobacteria, Acidobacteria, Actinobacteria, Firmicutes, Bacteroidetes, Chloroflexi, Gemmatimonadetes-were found to be predominating in general in soils of different countries [14,15,17-19]. The relatively high abundance of Firmicutes found in our study is instead controversially discussed in literature. Whereas Roesch et al. [14] listed Firmicutes in the prominent phyla present in soils, which is in agreement with our study, other authors analyzing tundra soil [15,18], grassland sites and other agricultural soils [17-19] did not describe this phylum as an highly abundant part of soil microbiomes.

Our main focus was on the effect of $\mathrm{N}$, which is an acknowledged key variable in microbial ecology. Cederlund and coworkers [6] demonstrated that $\mathrm{N}$ fertilization was the most important driver for changes in the relative abundances of soil bacteria and that no specific taxa were indicative of organic amendment in general.

Several authors reported, by comparing $\mathrm{N}$ fertilized soils with controls, shifts in the abundance of determined bacterial groups, such as Bacteroidetes, some members of Proteobacteria and Acidobacteria [17-19], Gemmatimonadetes and Verrucomicrobia [15]. Interestingly, in our study, Firmicutes were highly responding to the applied fertilizers as their apparent percentage increase was favored in the presence of fertilizers, such as Y, THL, CC or N. Urea-based fertilizers (UTY and 
UTN) did not show this positive effect on this phylum. As a possible explanation of this result, the presence of $\mathrm{N}$ in complex macromolecules, such as in organic fertilizers, could have enriched particular groups of organisms endowed with prompt adaptation to metabolize those (in this case, Firmicutes representing a competent heterotrophic group). Conversely, the presence of urea, which is an easy source of $\mathrm{N}$ for both plants and microorganisms, could have played a more neutral role, not favoring a specific phylum over others. For bacteria of the phylum Bacteroidetes, a higher relative abundance in the presence of $Y$ was observed, while bacteria of this phylum decreased in relative abundance when the fertilizer UTN was used. Bacteroidetes are considered copiotrophic ( $\mathrm{r}$-selected), and their increase in abundance in the presence of easily accessible $\mathrm{N}$ fertilization has already been reported [15,17-19]. Armatimonadetes and Nitrospirae, in contrast, appeared negatively affected by the $\mathrm{Y}$, which was expected as easily available $\mathrm{N}$ induces an out-competition mainly of the autotrophic microbes belonging to Nitrospirae [17]. In general, the presence of the $Y$ induced a decrease in abundance of many bacterial groups, favoring only a few abundant ones. In contrast, the presence of OATr fertilizer in soils brought about the relative increase of many low-abundant phyla. These proportional changes at the community level did not reflect significant differences in the calculated diversity indices. Results obtained by Fierer et al. [19] are in line with these observations. In fact, they detected no significant effects of $\mathrm{N}$ fertilization on bacterial diversity, but significant shifts in community composition. In contrast, other authors [18] found a decrease in bacterial diversity with $\mathrm{N}$ additions. These two contrasting visions suggest that the effects of $\mathrm{N}$ amendments on bacterial diversity levels within soil are variable and dependent on the site as well as the fertilizer quality, or even other effects such as changes in soil $\mathrm{pH}$ or micronutrient status.

Overall Bacteroidetes and Firmicutes, which were present at high relative abundances a few days after the starting of fertilization trial, showed a decrease in time in all soil samples. The majority of low-abundance phyla showed instead an increase of relative abundance over time, leading to a gain in evenness. Nevertheless, the analysis also allowed to differentiate effects for all applied fertilizers. For example, major ones were detected in response to the addition of the fertilizer $Y$, in particular at the beginning of the trial. Here, after 9 days, Firmicutes represented the $45 \%$ of the entire community, and decreased to $13 \%$ after two months. This situation could be explained by an initial rapid growth of this group of microorganisms in contact with $Y$, to the detriment of less abundant phyla that almost disappeared in this first phase. After about 60 days, the decrease in the relative abundance of Firmicutes could have caused the slight increase of the abundance of minor phyla and establishment of a more equilibrated condition. Soils treated with this organic fertilizer presented an apparent increase in the number of Proteobacteria over time, while, in other soils, this phylum remained stable.

It could be possible that the rapid changes observed soon after the addition of the treatments, apart from being due to the responses of bacteria already present in the substrate soil, could be in part also caused by a component of newly introduced microorganisms present in the fertilizers. However, as regards the influence of the bacteria carried by the fertilizers themselves, prior reports point out that their occurrence in soil does not appear to match their prevalence in the added substrates, as although Psychrobacter was the most abundant genus in pig manure, it was not detectable in the corresponding treated soil [7].

As a general comment, it is also worth recalling that, when analyzing these data, as inevitably the case in all metagenomics surveys, it needs to be kept in mind that they are based on percentage values and thus represent just relative abundances. Therefore, an apparent increase or decrease of a given taxon could not necessarily reflect a true active or passive dynamics, since these shifts could be the indirect effects of the behavior of other taxa.

\section{Conclusions}

Upon comparing the communities observed at day 9 in the unfertilized control soil with those of the soils having received the fertilizers at the same date (Figure 1 and Table S3), it can be observed that, in general, treatments caused a rapid change in the relative taxa abundances during the first 
days. The same comparisons relative to the later date (day 58) showed smaller differences between control and fertilized cases, suggesting a gradual stabilization of equilibrium with lower instances of dominance, which in turn would entail a rise of evenness. Such increase in evenness and its statistical significance was independently verified by a comparison between the two single time points by running the Wilcoxon test on the ecological indices.

The different shifts observed in soil bacteria suggest that $\mathrm{N}$ quality, in spite of $\mathrm{N}$ quantity, is the driver of community changes over time. This concurs to confirm the initial hypothesis: microbial communities responding differently according to the form in which the (constant) amount of $\mathrm{N}$ is supplied to them.

Particularly, evident was the effect of the fertilizer Y. This treatment caused a significant increase in plant yield as well a rapid and strong increase in the relative abundance of Firmicutes and Bacteroidetes, which in turn negatively influenced the share of less abundant phyla. Seeking for possible reasons, one could remark that Ys are complete microbial organisms and their extract is consequently rich in most life elements. While this could be beneficial for plants, as regards the effects on the growth of bacteria, it has also to be considered that those $Y$ biomasses come in part from antibiotics-producing bioreactors, which can in turn display negative impacts on given bacteria due to such antimicrobial product residuals. The other treatment having a peculiar and significant effect on bacteria was OATr, which was the only one causing a strong relative decrease in the number of Firmicutes and an increase in abundance of the low-number phyla. The two composite fertilizers UTY and UTN often caused similar changes at the phylum level, which were different from the effects caused by single component organic treatments. Future studies will be necessary to describe the consequences of the seemingly occurring shifts in bacterial community structure for the related functional traits as well as their expression profiles.

Supplementary Materials: The following are available online at http://www.mdpi.com/2571-8789/2/3/52/s1 within the document "Supplementary Material, Zanardo et al.": Figure S1A. Rarefaction analysis at the genus level, Figure S1B. Rarefaction analysis of the Shannon index; Table S1. Plant growth differences significance; Table S2. Number of genera detected across all samples within each phylum; Table S3. Genus level variation of taxa over time.

Author Contributions: Conceptualization, M.S., P.S., G.C., A.S.; investigation, M.Z., R.R., A.M., A.A., V.D., L.P., formal analysis, M.E., G.S., R.R.; funding acquisition: A.S., G.C., M.Z.; writing of the original draft preparation, A.S., M.S.

Funding: This work was supported in part by a PRAT 2012-CPDA127231 research grant from the University of Padova.

Acknowledgments: G.S. thanks the Department of Biodiversity and Molecular Ecology, Research and Innovation Centre, Fondazione Edmund Mach, San Michele all'Adige, Italy, for computational support.

Conflicts of Interest: The authors declare no conflicts of interest.

\section{References}

1. Schloss, P.D.; Handelsman, J. Toward a census of bacteria in soil. PLoS Comput. Biol. 2006, 2, e92. [CrossRef] [PubMed]

2. Gans, J.; Wolinsky, M.; Dunbar, J. Computational improvements reveal great bacterial diversity and high metal toxicity in soil. Science 2005, 309, 1387-1390. [CrossRef] [PubMed]

3. Goss-Souza, D.; Mendes, L.W.; Borges, C.D.; Baretta, D.; Tsai, S.M.; Rodrigues, J.L.M. Soil microbial community dynamics and assembly under long-term land use change. FEMS Microbiol. Ecol. 2017, 93. [CrossRef] [PubMed]

4. Szoboszlay, M.; Dohrmann, A.B.; Poeplau, C.; Don, A.; Tebbe, C.C. Impact of land-use change and soil organic carbon quality on microbial diversity in soils across Europe. FEMS Microbiol. Ecol. 2017, 93. [CrossRef] [PubMed]

5. Riber, L.; Poulsen, P.H.; Al-Soud, W.A.; Skov Hansen, L.B.; Bergmark, L.; Brejnrod, A.; Norman, A.; Hansen, L.H.; Magid, J.; Sørensen, S.J. Exploring the immediate and long-term impact on bacterial communities in soil amended with animal and urban organic waste fertilizers using pyrosequencing and screening for horizontal transfer of antibiotic resistance. FEMS Microbiol. Ecol. 2014, 90, 206-924. [CrossRef] [PubMed] 
6. Cederlund, H.; Wessén, E.; Enwalla, K.; Jones, C.M.; Juhanson, J.; Pell, M.; Philippot, L.; Hallin, S. Soil carbon quality and nitrogen fertilization structure bacterial communities with predictable responses of major bacterial phyla. Appl. Soil Ecol. 2014, 84, 62-68. [CrossRef]

7. Hamm, A.C.; Tenuta, M.; Krause, D.O.; Ominski, K.H.; Tkachuk, V.L.; Flaten, D.N. Bacterial communities of an agricultural soil amended with solid pig and dairy manures and urea fertilizer. Appl. Soil Ecol. 2016, 103, 61-71. [CrossRef]

8. Liu, P.; Jia, S.; He, X.; Zhang, X.; Ye, L. Different impacts of manure and chemical fertilizers on bacterial community structure and antibiotic resistance genes in arable soils. Chemosphere 2017, 188, 455-464. [CrossRef] [PubMed]

9. Tilman, D. Secondary succession and the pattern of plant dominance along experimental nitrogen gradients. Ecol. Monogr. 1987, 57, 189-214. [CrossRef]

10. Gough, L.; Osenberg, C.W.; Gross, K.L.; Collins, S.L. Fertilization effects on species density and primary productivity in herbaceous plant communities. OIKOS 2000, 89, 428-439. [CrossRef]

11. Vitousek, P.M.; Hattenschwiler, S.; Olander, L.; Allison, S. Nitrogen and nature. AMBIO 2002, 31, 97-101. [CrossRef] [PubMed]

12. Gilliam, F.S. Response of the herbaceous layer of forest ecosystems to excess nitrogen deposition. J. Ecol. 2006, 94, 1176-1191. [CrossRef]

13. Fierer, N.; Strickland, M.S.; Liptzin, D.; Bradford, M.A.; Cleveland, C.C. Global patterns in belowground communities. Ecol. Lett. 2009, 12, 1238-1249. [CrossRef] [PubMed]

14. Roesch, L.F.; Fulthorpe, R.R.; Riva, A.; Casella, G.; Hadwin, A.K.; Kent, A.D.; Daroub, S.H.; Camargo, F.A.; Farmerie, W.G.; Triplett, E.W. Pyrosequencing enumerates and contrasts soil microbial diversity. ISME J. 2007, 1, 283-290. [CrossRef] [PubMed]

15. Nemergut, D.R.; Townsend, A.R.; Sattin, S.R.; Freeman, K.R.; Fierer, N.; Neff, J.C.; Bowman, W.D.; Schadt, C.W.; Weintraub, M.N.; Schmidt, S.K. The effects of chronic nitrogen fertilization on alpine tundra soil microbial communities: Implications for carbon and nitrogen cycling. Environ. Microbiol. 2008, 10, 3093-3105. [CrossRef] [PubMed]

16. Acosta-Martínez, V.; Dowd, S.; Sun, Y.; Allen, V. Tag-encoded pyrosequencing analysis of bacterial diversity in a single soil type as affected by management and land use. Soil Biol. Biochem. 2008, 40, 2762-2770. [CrossRef]

17. Ramirez, K.S.; Lauber, C.L.; Knight, R.; Bradford, M.A.; Fierer, N. Consistent effects of nitrogen fertilization on soil bacterial communities in contrasting systems. Ecology 2010, 91, 3463-3470. [CrossRef] [PubMed]

18. Campbell, B.J.; Polson, S.W.; Hanson, T.E.; Mack, M.C.; Schuur, E.A. The effect of nutrient deposition on bacterial communities in Arctic tundra soil. Environ. Microbiol. 2010, 12, 1842-1854. [CrossRef] [PubMed]

19. Fierer, N.; Leff, J.W.; Adams, B.J.; Nielsen, U.N.; Bates, S.T.; Lauber, C.L.; Owens, S.; Gilbert, J.A.; Wall, D.H.; Caporaso, J.G. Cross-biome metagenomic analyses of soil microbial communities and their functional attributes. Proc. Natl. Acad. Sci. USA 2012, 109, 21390-21395. [CrossRef] [PubMed]

20. Zanardo, M.; Rosselli, R.; Meneghesso, A.; Sablok, G.; Stevanato, P.; Altissimo, A.; Peserico, L.; DeZuani, V.; Concheri, G.; Schloter, M.; et al. Dynamics of soil prokaryotes catalyzing nitrification and denitrification in response to different fertilizers in a greenhouse experiment with Cynodon dactylon. Eur. J. Soil Biol. 2016, 76, 83-91. [CrossRef]

21. Pilloni, G.; Granitsiotis, M.S.; Engel, M.; Lueders, T. Testing the limits of 454 pyrotag sequencing: Reproducibility, quantitative assessment and comparison to T-RFLP fingerprinting of aquifer microbes. PLoS ONE 2012, 7, e40467. [CrossRef] [PubMed]

22. Berry, D.; Mahfoudh, K.B.; Wagner, M.; Loy, A. Barcoded primers used in multiplex amplicon pyrosequencing bias amplification. Appl. Environ. Microbiol. 2011, 77, 7846-7849. [CrossRef] [PubMed]

23. Caporaso, J.G.; Kuczynski, J.; Stombaugh, J.; Bittinger, K.; Bushman, F.D.; Costello, E.K.; Fierer, N.; Peña, A.G.; Goodrich, J.K.; Gordon, J.I.; et al. QIIME allows analysis of high-throughput community sequencing data. Nat. Methods 2010, 7, 335-336. [CrossRef] [PubMed]

(c) 2018 by the authors. Licensee MDPI, Basel, Switzerland. This article is an open access article distributed under the terms and conditions of the Creative Commons Attribution (CC BY) license (http:/ / creativecommons.org/licenses/by/4.0/). 